\title{
Mission : Avoid Antibiotic Abuse
}

\author{
VIJAY N YeWALE \\ National President, Indian Academy of Pediatrics, 2014. \\ vnyewale@gmail.com
}

$\mathrm{F}$ ostering awareness about antimicrobial resistance and rational antibiotic practice is a part of the IAP ICMR Call to Action declared this year. And it is action time now!

As a commitment to reduce antibiotic misuse, Septemer 28 will be observed as the Rational Antibiotic Day and the week thereafter, has been declared as Antibiotic Awareness Week. September 28 has been selected in commemoration with Sir Alexander Flemming's discovery of penicillin. This week culminates in a first national conference exclusively on antimicrobials - Antimicrobiocon 2014 - at Ahmedabad on October 4-5, 2014.

The slogan and poster competition received a good response from the postgraduate students of medical institutes. The various branches of IAP are geared up for the week long activities which include display of banners, distribution of leaflets, meeting with pharmaceutical representatives to discourage promotion of irrational combinations, walk/rallies for antibiotic awareness, talks in media, webinars, and sensitizing the family practitioners on rational antibiotic practices through lectures/CMEs. Indian Medical Association has joined the mission and will help reach all sections of medical professionals.

Even if the onus of prescribing antibiotic rationally lies with the medical practitioners, it is equally important that the medicines are administered appropriately by the caretaker/patient. There is high practice of self medication of antibiotics, especially due to easy availability of antibiotics over-the-counter. Ignorance about completing the course in proper dosages leads to incomplete treatment. Lack of knowledge about dilution and reconstitution of antibiotic also hampers complete therapy. It is time that patients are made aware so that they start questioning the doctors on why an antibiotic has been prescribed, and if it is really needed. It will certainly deter the prescriber from misusing antibiotic. All our members need to start writing clinical diagnosis on paper. This will make them think before they ink an antibiotic as a routine reflex. The chemists may also be instructed not to dispense an antibiotic unless a provisional diagnosis or clinical diagnosis is mentioned on the prescription.

A survey revealed that $35 \%$ of the physicians felt moderate, and $11 \%$ a strong pressure to prescribe antibiotics because of patient requests or expectations [1]. In another survey [2], many doctors felt that prescribing the latest and expensive antibiotics not only removed their insecurity about making correct diagnosis but the patients were satisfied that their visit had been fruitful. Moreover, many patients either demanded the same prescription for a recurrent episode of similar symptoms or used the same old prescription to save time and money.

The need of the hour is therefore to involve the general public to join hands with the health professionals to overcome this challenge of curbing antibiotic resistance. It was shown by Belgian researchers that antibiotic usage dropped by $11.7 \%$ during a flu-like outbreak that occurred after a public awareness campaign stressing the proper use of antibiotics [3]. The awareness about the gravity of antibiotic resistance, and that antibiotics are not the cure for every cold, cough or diarrhea, will help significantly in both reducing the pressure on doctors for antibiotics and also promote judicious consumption with respect to dosage and duration. Several such campaigns are being regularly organized across countries like Europe, US and Canada. Such campaigns are of paramount importance in our country where the infectious disease burden is largest.

\section{REFERENCES}

1. Sivagnanam G, Mohanasundaram J, Thirumalaikolundusubramanian P, Raaj A, Namasivayam K, Rajaram S. A survey on current attitude of practicing physicians upon usage of antimicrobial agents in Southern part of India. MedGenMed. 2004;6:1.

2. Kotwani A, Wattal C, Katewa S, Joshi PC, Holloway K. Factors influencing primary care physicians to prescribe antibiotics in Delhi India. Fam Pract. 2010;27:684-90.

3. Bauraind I, Lopez-Lozano JM, Beyaert A, Marchal JL, Seys $\mathrm{B}$, Yane F, et al. Association between antibiotic sales and public campaigns for their appropriate use. JAMA. 2004;292:2468-70. 